\title{
Elf5-centered transcription factor hub controls trophoblast stem cell self-renewal and differentiation through stoichiometry- sensitive shifts in target gene networks
}

\author{
Paulina A. Latos, ${ }^{1,2}$ Arnold R. Sienerth, ${ }^{1,2}$ Alexander Murray, ${ }^{1,2}$ Claire E. Senner, ${ }^{1,2}$ Masanaga Muto, ${ }^{3}$ \\ Masahito Ikawa, ${ }^{3}$ David Oxley, ${ }^{4}$ Sarah Burge, ${ }^{1}$ Brian J. Cox, ${ }^{5,6}$ and Myriam Hemberger ${ }^{1,2}$ \\ ${ }^{1}$ Epigenetics Programme, The Babraham Institute, Cambridge CB22 3AT, United Kingdom; ${ }^{2}$ Centre for Trophoblast Research, \\ University of Cambridge, Cambridge CB2 3EG, United Kingdom; ${ }^{3}$ Graduate School of Pharmaceutical Sciences, Animal Resource \\ Center for Infectious Diseases, Research Institute for Microbial Diseases, Osaka University, Suita, Osaka 565-0871, Japan; \\ ${ }^{4}$ Proteomics Group, The Babraham Institute, Cambridge CB22 3AT, United Kingdom; ${ }^{5}$ Department of Physiology, Faculty of \\ Medicine, University of Toronto, Toronto, Ontario M5S 1A8, Canada; ${ }^{6}$ Department of Obstetrics and Gynaecology, Faculty of \\ Medicine, University of Toronto, Toronto, Ontario M5G 1E2, Canada
}

Elf5 is a transcription factor with pivotal roles in the trophoblast compartment, where it reinforces a trophoblast stem cell (TSC)-specific transcriptional circuit. However, Elf5 is also present in differentiating trophoblast cells that have ceased to express other TSC genes such as Cdx2 and Eomes. In the present study, we aimed to elucidate the context-dependent role of Elf5 at the interface between TSC self-renewal and the onset of differentiation. We demonstrate that precise levels of Elf5 are critical for normal expansion of the TSC compartment and embryonic survival, as Elf5 overexpression triggers precocious trophoblast differentiation. Through integration of protein interactome, transcriptome, and genome-wide chromatin immunoprecipitation data, we reveal that this abundancedependent function is mediated through a shift in preferred Elf5-binding partners; in TSCs, Elf5 interaction with Eomes recruits Tfap2c to triply occupied sites at TSC-specific genes, driving their expression. In contrast, the Elf5 and Tfap2c interaction becomes predominant as their protein levels increase. This triggers binding to double- and single-occupancy sites that harbor the cognate Tfap2c motif, causing activation of the associated differentiationpromoting genes. These data place Elf5 at the center of a stoichiometry-sensitive transcriptional network, where it acts as a molecular switch governing the balance between TSC proliferation and differentiation.

[Keywords: trophoblast stem cells; transcriptional networks; transcription factor complexes; self-renewal; control of differentiation]

Supplemental material is available for this article.

Received July 15, 2015; revised version accepted October 30, 2015.

The repertoire of transcription factors (TFs) expressed in any given cell is a critical determinant of cellular identity and developmental capacity. As such, TFs are pivotal in regulating the self-renewal and differentiation potential of stem cells. Much of our knowledge in this area has come from the analysis of embryonic stem cells (ESCs) as well as induced pluripotent stem cells (iPSCs). This work has elucidated a core set of TFs-namely, Oct4 and Sox2-that is essential for pluripotency and self-renewal, in addition to numerous "accessory" factors that help promote naïve pluripotency (Nichols and Smith 2012; Martello and Smith 2014).

Corresponding authors: myriam.hemberger@babraham.ac.uk, b.cox@ utorono.ca

Article published online ahead of print. Article and publication date are online at http://www.genesdev.org/cgi/doi/10.1101/gad.268821.115. Freely available online through the Genes \& Development Open Access option.
Besides TF identity and combination, defined protein levels and the stoichiometry of TFs in relation to each other influence the balance between stem cell self-renewal and differentiation. In ESCs, this concept is evidenced by Oct4, as either constitutively high expression levels or its down-regulation result in destabilization of the pluripotent state and differentiation (Niwa et al. 2000; Radzisheuskaya et al. 2013). Relative abundance of Oct4 in proportion to Cdx2 determines embryonic versus trophoblast lineage fate in ESCs (Niwa et al. 2005). Similarly, the stoichiometry of reprogramming factors influences the biological properties of iPSCs (Carey et al. 2011).

(C) 2015 Latos et al. This article, published in Genes \& Development, is available under a Creative Commons License (Attribution-NonCommercial 4.0 International), as described at http://creativecommons.org/ licenses/by-nc/4.0/. 
While the regulatory nature of TF stoichiometry has been explored in ESCs and iPSCs, it is not known whether similar mechanisms of control over self-renewal and differentiation underpin the behavior of stem cells of the trophoblast lineage. Trophoblast stem cells (TSCs) are a stem cell type representative of the extraembryonic placental lineage most akin to cells in the extraembryonic ectoderm (ExE), a structure that eventually gives rise to specialized placental cell types (Tanaka et al. 1998; Adachi et al. 2013). TSCs can self-renew and remain multipotent in the presence of fibroblast growth factor (Fgf) and embryonic fibroblast-conditioned medium (CM). Upon withdrawal of Fgf and CM, TSCs differentiate into various trophoblast cell types of the chorioallantoic placenta (Tanaka et al. 1998). A number of TFs are known to be involved in either the determination of trophoblast cell fate and/or the ability to derive or maintain TSCs. Among these are the TEA domain family member Tead4 (Yagi et al. 2007; Nishioka et al. 2008), the caudal-type homeobox factor Cdx2, the Tbox gene Eomes, the SRY-box gene Sox2, and the estrogen-related receptor Esrrb (Russ et al. 2000; Tremblay et al. 2001; Avilion et al. 2003; Strumpf et al. 2005; Ralston and Rossant 2008; Adachi et al. 2013; Latos et al. 2015). Other TFs important for the establishment of a self-renewing TSC population include the AP-2 family member Tfap2c (Auman et al. 2002; Werling and Schorle 2002), the Ets family members Ets2 and Elf5 (Yamamoto et al. 1998; Donnison et al. 2005), and the Gata motif-containing factor Gata3 (Home et al. 2009; Ralston et al. 2010). As all of these TFs are expressed in both ExE and TSCs, the latter provide an excellent in vitro model to study the trophoblast TF networks during self-renewal and the onset of differentiation.

Genome-wide occupancy analyses of Cdx2, Elf5, Eomes, Ets2, Tfap2c, Gata3, Sox2, and Esrrb in TSCs revealed that they cobind a significant number of target loci, including themselves, to establish a self-reinforcing TSC-specific transcriptional network (Kidder and Palmer 2010; Adachi et al. 2013; Chuong et al. 2013; Latos et al. 2015). However, despite coexpression in the TSC compartment in vivo and in vitro, the precise temporal and spatial expression domains of these various TFs are not fully overlapping. For instance, Cdx2 and Elf5 are coexpressed in TSCs, but, upon induction of differentiation, $\mathrm{Cdx} 2$ is down-regulated more rapidly than Elf5 (Ng et al. 2008). Similarly in vivo, Cdx2 and Eomes are restricted to a narrow region of the ExE immediately overlying the epiblast, whereas Elf5 and Tfap2c have a much broader expression domain extending into the proximal ExE and ectoplacental cone (EPC) (Auman et al. 2002; Ng et al. 2008; Ralston and Rossant 2008; Kuckenberg et al. 2010; Adachi et al. 2013). These data point to a seeming discrepancy: Although core TFs are coexpressed and can mutually activate each other in the TSC compartment, their expression domains become divergent with the onset of differentiation. This paradox prompted us to speculate whether the precise function of a TF in different trophoblast compartments depends on its stoichiometric abundance in relation to other TFs.
In the present study, we hypothesized that Elf5 has a context-dependent role at the interface between TSC self-renewal and differentiation. We addressed this question by manipulating Elf5 expression levels in vitro and in vivo and thereby demonstrate that precise amounts of Elf5 are critical for TSC maintenance, normal placentation, and embryonic survival. By determining the Elf5 protein interaction networks and integrating them with gene expression as well as TF-based chromatin immunoprecipitation (ChIP) combined with deep sequencing (ChIP-seq) profiles, we reveal that Elf5 controls the balance between TSC self-renewal and differentiation through stoichiometry-sensitive interactions with Eomes and Tfap2c. This stoichiometry-dependent composition of TF complexes determines their genomic distribution, activating either TSC or differentiation-associated genes, and provides a mechanistic explanation of how self-renewal factors can also drive the exit from the stem cell compartment.

\section{Results}

\section{Elf5 levels are critical for the establishment of a proliferative TSC compartment}

Despite the mutual coactivation capacity of the three core TSC TFs Cdx2, Eomes, and Elf5, previous evidence showed that their expression patterns do not fully overlap (Ng et al. 2008). In outgrowths of trophoblast tissue, it was observed that the central, most TSC-like cells are largely double positive for Cdx2 and Elf5, while the surrounding, flatter cells still retain Elf5 but have largely lost Cdx2 (Supplemental Fig. S1A). Quantification of immunostaining signals further indicated that these Cdx2-low cells exhibited overall higher levels of Elf5 protein than the central stem-like population (Supplemental Fig. S1B,C). These data prompted us to hypothesize that the precise quantities of Elf5 may be important for TSC maintenance and that increased Elf5 amounts may trigger the exit from the proliferative TSC niche and prime trophoblast cells to differentiate.

To test this hypothesis in a physiologically meaningful way, we generated Elf5 and vector (GFP) control transgenic conceptuses by lentiviral transduction of blastocysts, thereby driving trophoblast-specific Elf5 (or GFP) overexpression (Fig. 1A; Supplemental Fig. S1D; Georgiades et al. 2007; Okada et al. 2007). When dissected at embryonic day 8.5 (E8.5) and E10.5, histological analysis revealed severe abnormalities in many Elf5 transgenic conceptuses (Fig. 1B). Already at E8.5, 50\% of Elf5 transgenic implantation sites were significantly smaller than GFP-positive wild-type controls and exhibited a proportional overabundance of proliferin-positive $($ Prl2c2 $=$ Plf $)$ trophoblast giant cells at the expense of diploid Tpbpa-expressing spongiotrophoblast precursors (Fig. 1C). In the remaining E8.5 conceptuses, milder defects were obvious in that the typically very columnar and regularly stacked E-Cadherin (Cdh1)-positive trophoblast cells at the base of the chorion appeared less well organized and had a more rounded morphology, indicative of epithelial integrity defects and precocious differentiation (Fig. 1D). To gain more detailed insights into the precise effects of Elf5 
A
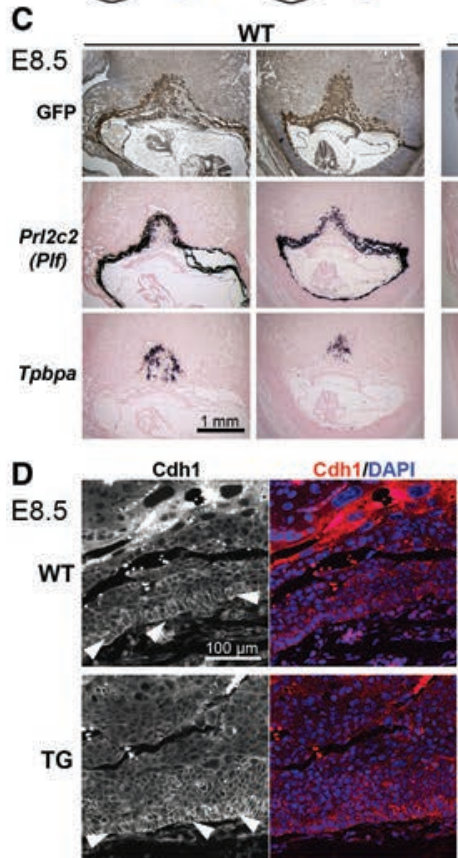

F10.5

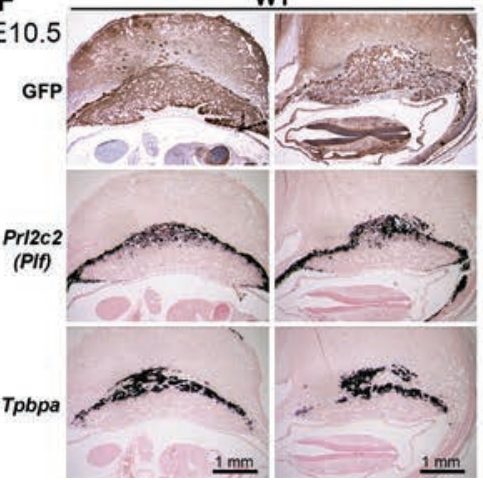

B

WT
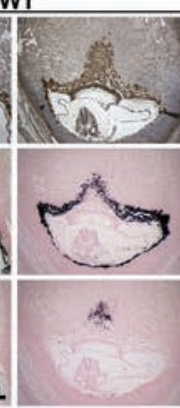
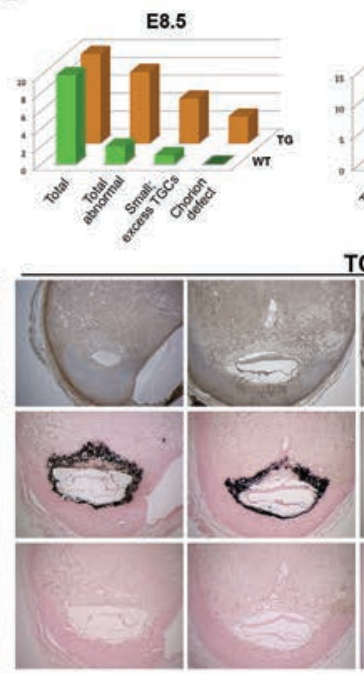

TG
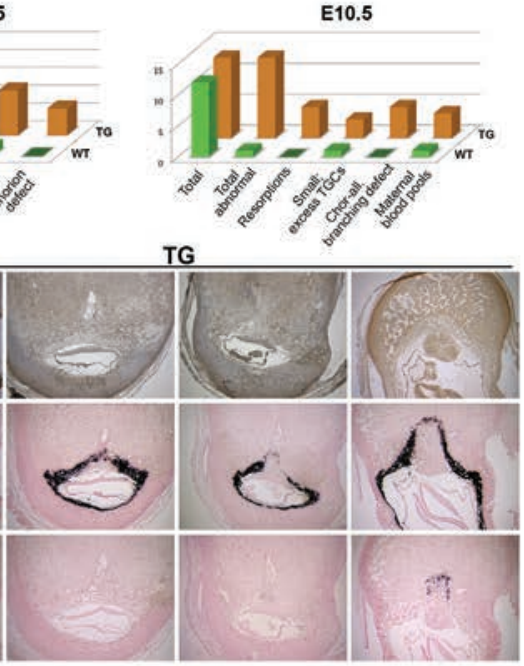

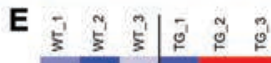

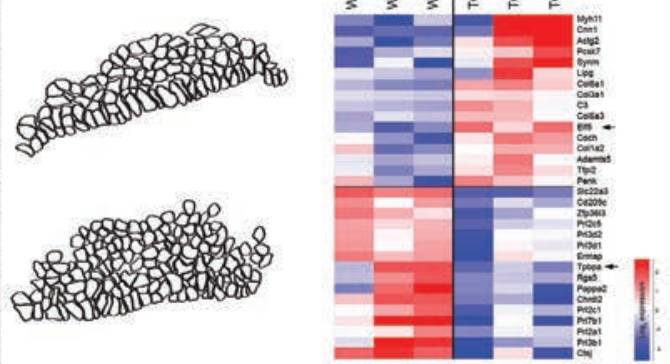

TG

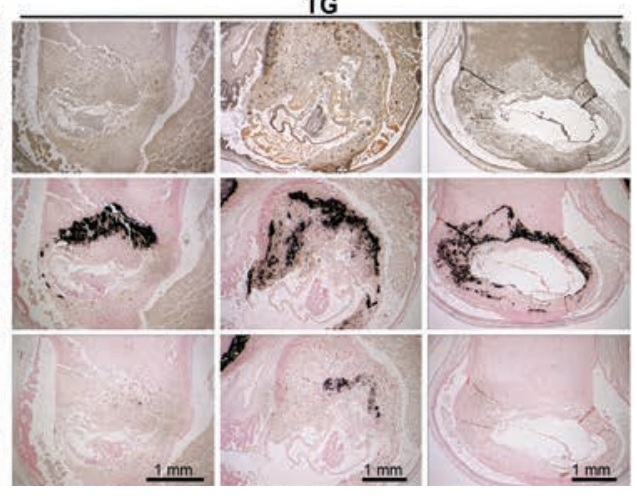

Figure 1. Elevated Elf5 levels cause premature trophoblast differentiation and embryonic lethality around mid-gestation. (A) Schematic diagram of lentiviral transduction experiments to confer trophoblast-specific overexpression of Elf5 (transgenic [TG]) or a GFP (wild-type [WT]) vector control construct. (B) Summary of developmental defects observed at E8.5 and E10.5. At E8.5, eight out of 10 Elf5 transgenic conceptuses exhibited developmental abnormalities; at E10.5, all 13 of 13 conceptuses were abnormal and often already in the process of resorption. (C) Histological analysis of E8.5 implantation sites. Immunohistochemistry against GFP identified control transduced, wild-type conceptuses exhibiting trophoblast-specific staining. Transgenic implantation sites were often smaller and consistently exhibited fewer diploid EPC trophoblast cells, demarcated by Tpbpa expression, but proportionately more giant cells, marked by Prl2c2 (also known as Plf). $(D)$ Immunofluorescence staining for E-Cadherin (Cdh1) on a wild-type and transgenic conceptus that still appeared grossly normal at this stage (E8.5). The tight arrangement of cuboidal chorionic trophoblast cells was less well organized (arrowheads) in all transgenic conceptuses analyzed. $n=3$. For clarification, the outlines of cells were drawn from the immunostaining and are depicted separately. (E) RNA-seq analysis from trophoblast tissue material recovered from paraffin sections of three wild-type and three transgenic conceptuses. The heat map displays consistently deregulated loci that include a number of genes involved in cytoskeletal organization, growth factor availability, and signal transduction cascades. $(F)$ Histological analysis of wild-type and transgenic conceptuses at E10.5. All transgenic implantation sites exhibit severe developmental abnormalities and are characterized by an overabundance of trophoblast giant cells (Plf) and the frequent appearance of vast maternal blood pools, indicative of a starting resorption process.

overexpression at these early stages, we compared global expression profiles (RNA sequencing [RNA-seq]) of chorionic trophoblast tissue recovered from these E8.5 paraffinembedded sections, thus allowing us to directly correlate histological appearance and transcriptomic changes. This analysis confirmed up-regulation of Elf5 and down-regulation of Tpbpa in transgenic trophoblasts, as expected (Fig. 1E). It also revealed misregulation of a number of genes involved in cytoskeletal and extracellular matrix organization (e.g., Actg2, Col1a2, Col3a1, Colba1, Col6a3, and Myh11), growth factor availability (Chrdl2 and Pappa2), and angiogenesis (Rgs5), as reflected by gene ontology enrichments (Supplemental Fig. S1E). These expression changes underpin the cellular dysmorphology phenotype at the molecular level.
At E10.5, transgenic implantation sites exhibited a vast overabundance of trophoblast giant cells and were often infiltrated by leukocytes, indicative of a starting resorption process (Fig. 1F). When embryos were dissected at E13.5, only control conceptuses exhibiting placental GFP signals were retrieved (Table 1). Taken together, these data demonstrated that Elf5 overexpression causes precocious trophoblast differentiation, leading to embryonic lethality around mid-gestation.

\section{Elf5 protein interactome}

Having shown that Elf5 levels are critical for establishing a proliferative TSC compartment in vivo, we aimed to identify the protein interaction partners of Elf5 that 
Table 1. Summary of transgenic conceptuses transferred and retrieved after lentiviral transduction

\begin{tabular}{lccccc}
\hline & \multicolumn{2}{c}{ Wild-type $\left(\mathrm{GFP}^{+}\right)$} & & \multicolumn{2}{c}{ Transgenic $\left(\mathrm{Elf5}^{+}\right)$} \\
\cline { 2 - 3 } \cline { 5 - 6 } & Transferred & Retrieved & & Transferred & Retrieved \\
\hline E8.5 & 10 & 10 & & 10 & 10 \\
E10.5 & 12 & 12 & & 13 & 13 \\
E13.5 & 12 & 12 & & 12 & 0 \\
\hline
\end{tabular}

contribute to maintaining a self-renewal network in TSCs and may be sensitive to relative changes in its protein quantities. For this purpose, we established TSC lines stably expressing a 3xFlag-tagged Elf5 construct at low levels (Supplemental Fig. S2A,B). These lines were used for protein immunoprecipitation (Supplemental Fig. S2C) followed by mass spectrometry (van den Berg et al. 2010) to identify Elf5-binding partners in an unbiased manner. Among these, we found factors implicated in trophoblast function, such as the transcriptional regulators Bptf (Goller et al. 2008) and Grhl2 (Walentin et al. 2015); Sall4, a TF better known for its role in ESC pluripotency (Zhang et al. 2006; Yang et al. 2008); and components of epigenetic-repressive and nucleosome remodeling complexes (Suz12, Sin3a, and Smca5) (Fig. 2A). Interestingly, we also identified several members of the Integrator complex, thus supporting a previous study suggesting that this complex may form a key part of the basal transcriptional machinery in TSCs (Latos et al. 2015).

Most importantly, with our focus specifically on TFs with known functions in TSCs, we noted Eomes and Tfap2c among the top-ranking high-confidence interactors, both well known for their role in trophoblast development and TSC maintenance (Fig. 2A; Supplemental Table S1). Elf5 binding to Eomes and Tfap2c was independently verified by coimmunoprecipitation of Flag-tagged Elf5 followed by Western blotting (Fig. 2B,C; Supplemental Fig. S2D,E). Moreover, we confirmed protein-protein interactions between all three TFs in reciprocal immunoprecipitations using Flag-tagged Eomes as well as Tfap2c as bait (Fig. 2D,E). Overall, these experiments established the Elf5 protein interactome in TSCs, providing significant insights into its function in the trophoblast compartment and demonstrating direct protein-protein interactions between the three key trophoblast TFs Elf5, Eomes, and Tfap2c.

\section{Dynamics of the Elf5, Eomes, and Tfap2c 'troika'}

Identification of these Elf5-centered $\mathrm{TF}$ complexes prompted us to characterize the precise expression dynamics of Eomes, Elf5, and Tfap2c. In differentiation timecourse experiments in vitro, Eomes expression was rapidly down-regulated. In contrast, Elf5 levels briefly peaked within the first $12 \mathrm{~h}$ of differentiation (Donnison et al. 2015) before gradually declining, albeit at a slower pace than Eomes (Fig. 3A,B). Tfap2c expression was present in TSCs, but its levels increased with differentiation. On the cellular level, these changes in relative protein quanti- ties were equally obvious, as onset of differentiation was associated with a rapid decline of Eomes and concomitant increase in Tfap2c (Fig. 3C).

These shifts in relative TF abundance were also evident in vivo; thus, the presumptive TSC progenitor compartment of the E6.5 ExE directly overlying the epiblast was positive for all three TFs-Eomes Elf5, and Tfap2c. However, as cells progressed toward the EPC, Eomes expression was abruptly lost, and Elf5 expression persisted and increased moderately, while Tfap2c expression was significantly up-regulated (Fig. 3D; Supplemental Fig. S2F). Trophoblast giant cells were negative for Elf5 but still expressed Tfap2c (data not shown). These data suggest that in vivo Elf5 down-regulation is more protracted, likely as these cells are exposed to a sustained Fgf4 signal (Shimokawa et al. 2011), in contrast to the immediate and complete withdrawal of Fgf in culture. However, the overall dynamics of TF regulation are mirrored in vivo and in vitro, insofar as Eomes is specific to the TSC compartment, whereas Elf5 and Tfap2c are present at even higher levels also in differentiating cells (Fig. 3E).

To determine whether this change in abundance of Eomes and Tfap2c relative to Ef15 impacts on its preferred binding partner, we performed Elf5 immunoprecipitations from TSCs grown in stem cell conditions and upon $1 \mathrm{~d}$ of differentiation and tested for relative enrichment of Eomes and Tfap2c (Fig. 3F). We also determined the precise amounts of Elf5 interaction partners by mass spectrometric quantification (Fig. 3G; Supplemental Fig. S2G). Intriguingly, enrichment of Eomes declined sharply, whereas Elf5 interaction with Tfap2c was preferred with the onset of differentiation (Fig. 3F,G). Other proteins identified as interacting with Elf5 did not change in enrichment levels between these two stages and served as internal controls. These data demonstrated that Elf5 is positioned at the fulcrum between stem cell maintenance and differentiation through preferential binding to Eomes and Tfap $2 \mathrm{c}$, respectively.

\section{Stoichiometry-dependent effects of Eomes, Elf5, and Tfap2c}

To pinpoint the effects of relative levels of Eomes, Elf5 and Tfap2c, we generated multiple constructs conferring expression of each of these TFs individually and in combination with Elf5 (Fig. 4A; Supplemental Fig. S3). These constructs were transfected into TSCs, and the presence of the expected proteins was confirmed (Supplemental Fig. S3A,F). Consistently, short-term (Fig. 4B) and longterm (Fig. 4C,D; Supplemental Fig. S3H,I) overexpression of Elf5 and Tfap2c, either alone or in combination, promoted TSC differentiation, most significantly toward trophoblast giant cells and similar in extent to early stage differentiation induced by Fgf and CM withdrawal (Supplemental Fig. S3B). The prodifferentiation effect was even more pronounced upon single-cell cloning, when most Elf5-, Tfap2c-, and Tfap2c_Elf5-overexpressing cells stopped proliferating and exhibited clear morphological features of differentiation (Supplemental Fig. S3C). Only cells overexpressing very low levels of these two TFs 


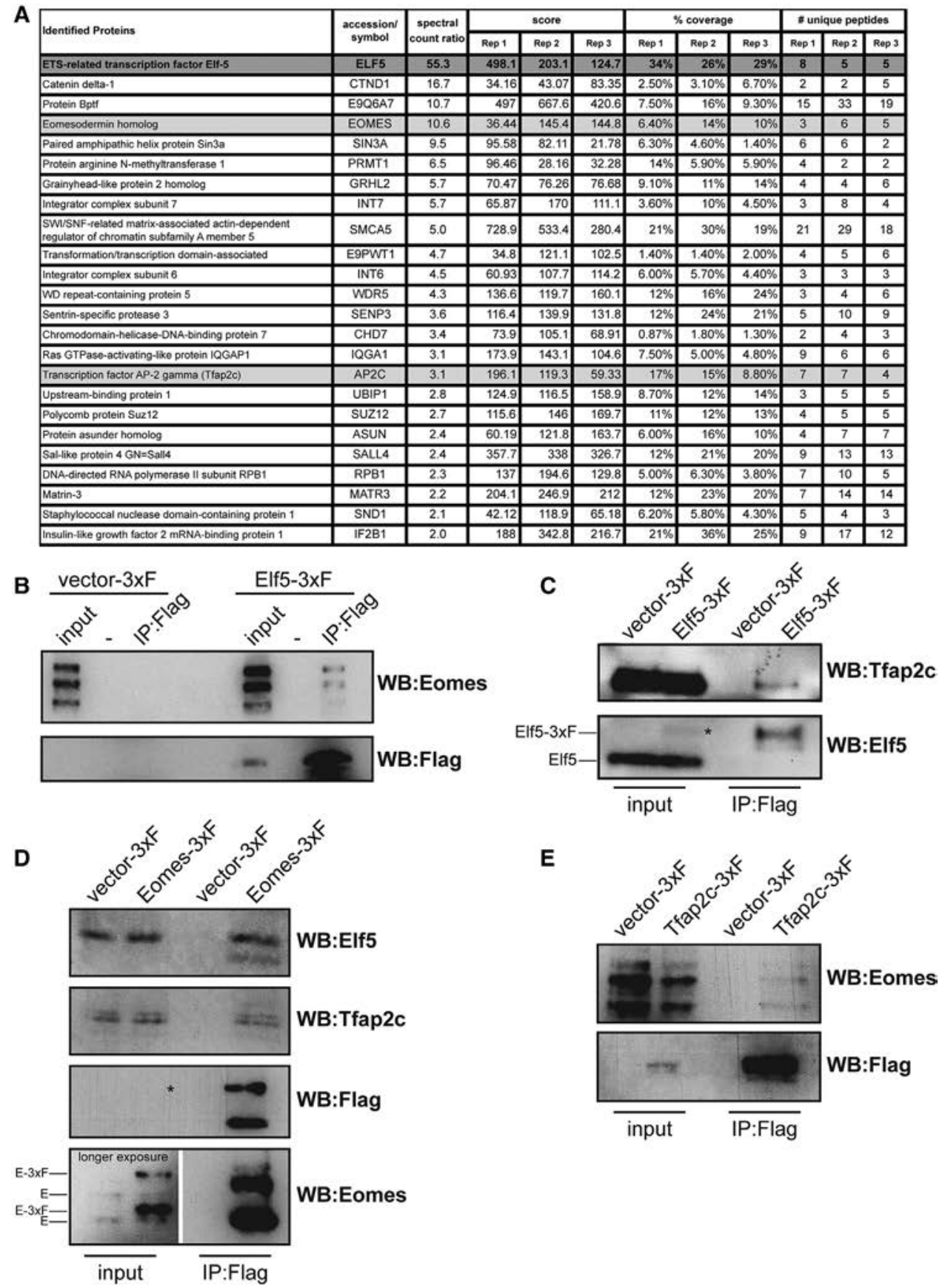

Figure 2. Identification of the Elf5 protein interactome in TSCs. (A) Summary of high-confidence Elf5 interactors as identified in three independent immunoprecipitation (IP) and mass spectrometry experiments. Note the interaction of Elf5 with the two other TSC TFs: Eomes and Tfap2c. The full list of interactors is provided in Supplemental Table S1. (B) Independent validation of the interaction between Elf5 and Eomes by anti-Flag immunoprecipitation of the $3 x$ Flag-tagged $(3 \mathrm{xF})$ Elf5 protein followed by Western blotting. (C) Independent validation of the interaction between Elf5 and Tfap2c by immunoprecipitation followed by Western blotting. The asterisk highlights the Elf5-3xFlag band in the input sample. Additional validation of the Elf5-Tfap2c interaction is provided in Figure 3F and Supplemental Figure S2, D and E. (D) Eomes interacts with Tfap2c and Elf5. Eomes-3xFlag immunoprecipitates analyzed by Western blot using anti-Elf5 and anti-Tfap2c antibodies. The asterisk demarcates the presence of Eomes in the input samples that can be appreciated upon longer exposure, shown in the panel below. (E) Tfap2c interacts with Eomes. Tfap2c-3xFlag immunoprecipitates analyzed by Western blot using anti-Eomes antibody.

were capable of forming colonies and could be propagated long-term (Fig. 4E; Supplemental Fig. S3D,G). These data were entirely in line with the Elf5 overexpression phenotype that we observed in vivo (Fig. 1).
In contrast, Eomes-overexpressing cells exhibited an undifferentiated, epithelial morphology and a TSClike gene expression pattern (Fig. 4C,D; Supplemental Fig. S3H,I). Significantly, the Elf5- and Tfap2c-induced 

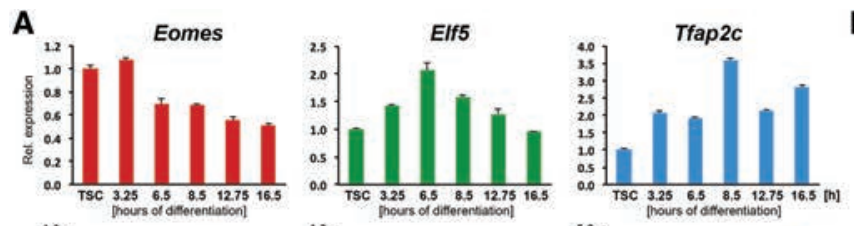

B
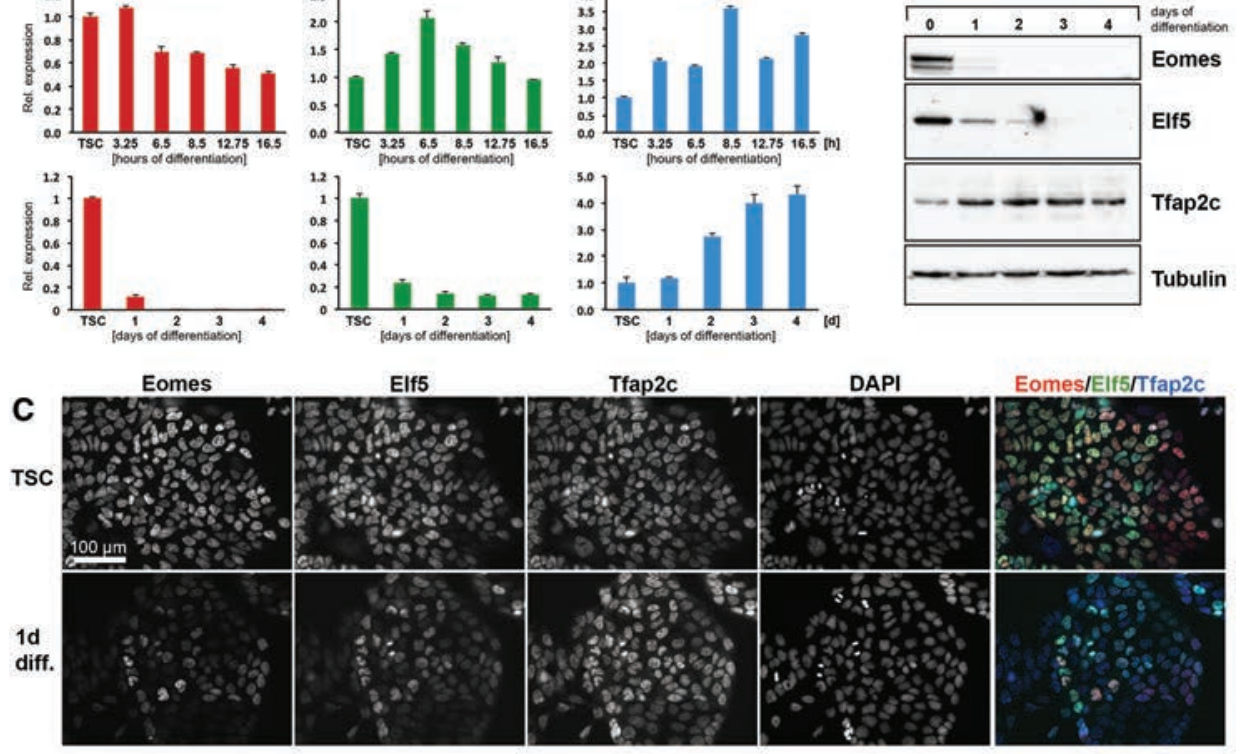

D
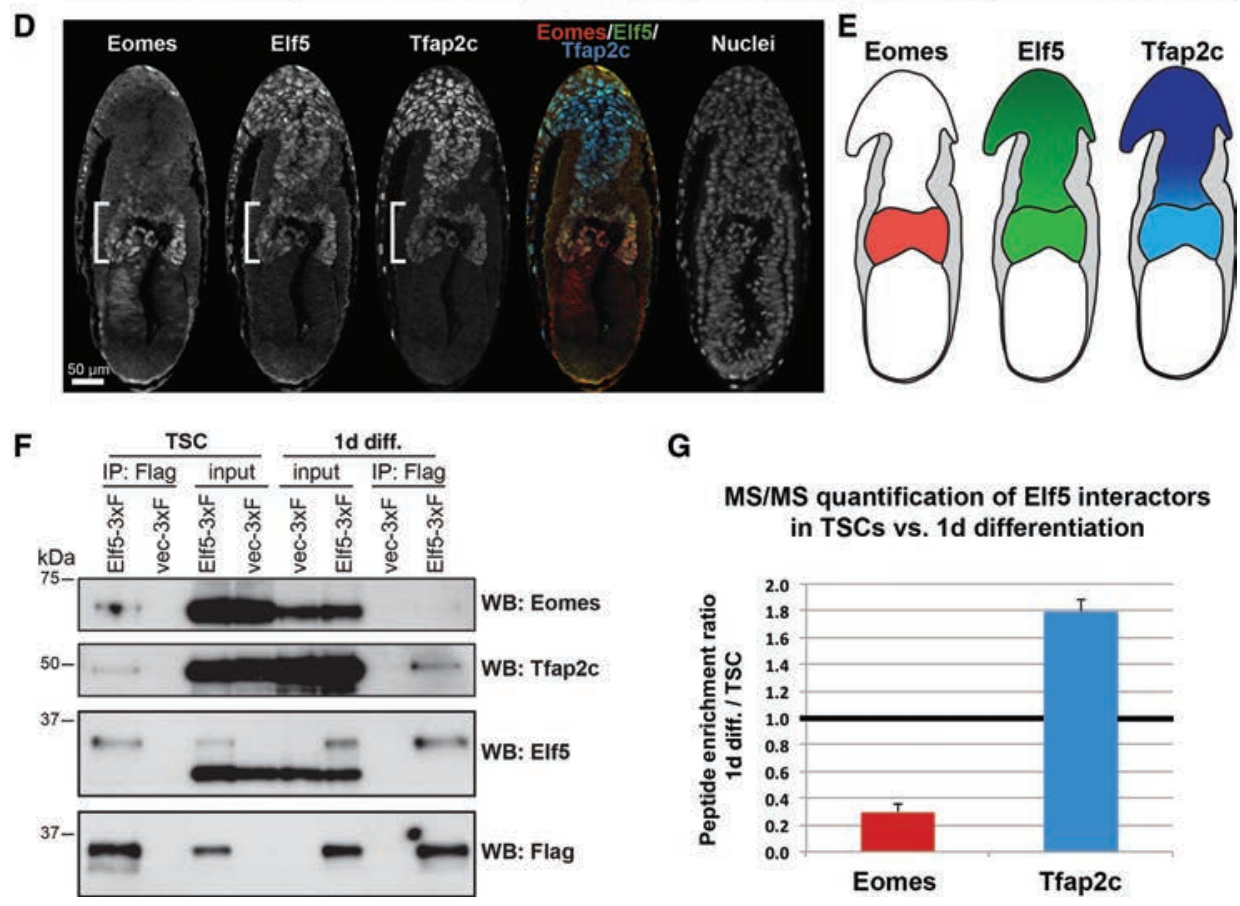

G

\section{MS/MS quantification of Elf5 interactors in TSCs vs. 1d differentiation}

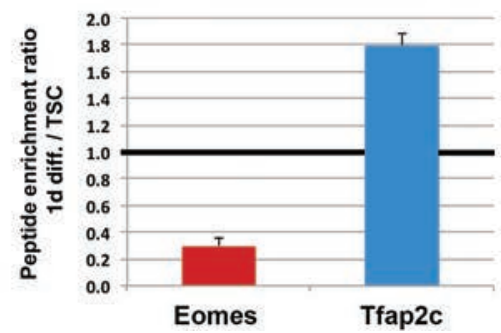

Figure 3. Dynamics of Elf5, Eomes, and Tfap2c expression. (A) Expression dynamics of Eomes, Elf5, and Tfap2c by RT-qPCR in shortterm (top row) and longer-term (bottom row) differentiation time-course experiments of TSCs. $(B)$ Western blots confirming the dynamic regulation of Eomes, Elf5, and Tfap2c upon trophoblast differentiation. $(C)$ Immunofluorescence stainings of TSCs grown in stem cell conditions (TSC) and after $1 \mathrm{~d}$ of differentiation (1 d diff.) for Eomes, Elf5, and Tfap2c. (D) Immunofluorescence stainings of E6.5 conceptuses for Eomes, Elf5, and Tfap2c. All three TFs are expressed in the ExE region that harbors trophoblast cells with stem cell potential (bracket). Elf5 and Tfap2c expression is retained in trophoblast cells outside this compartment that start to differentiate. $(E)$ Schematic representation of the comparative expression patterns and relative protein levels (darker shading indicates higher expression levels) of Eomes, Elf5 and Tfap2c in the trophoblast compartment of early post-implantation conceptuses. (F) Immunoprecipitation (IP) of 3x-Flag-tagged (3xF) Elf5 and empty vector (vec) control from TSCs and 1-d (24-h)-differentiated TSCs followed by Western blotting for the factors indicated. Note the higher enrichment of Eomes in Elf5 immunoprecipitations from TSCs versus differentiated cells. Conversely, Tfap2c is more abundant in Elf5 immunoprecipitations from differentiated trophoblasts. The Western blot for Elf5 confirms the presence of the tagged Elf5-3xFlag protein in the corresponding cell lines that is of higher molecular weight than the endogenous Elf5 present in the input samples. $(G)$ Mass spectrometric quantification of Eomes and Tfap2c peptide enrichment in Elf5-3xFlag immunoprecipitations. Values were normalized against Hspa8 that did not change enrichment with differentiation. Results show the significant decrease in Eomes and increase in Tfap2c binding to Elf5 upon TSC differentiation and are representative of two independent immunoprecipitation-tandem mass spectrometry (MS/MS) experiments. 
A

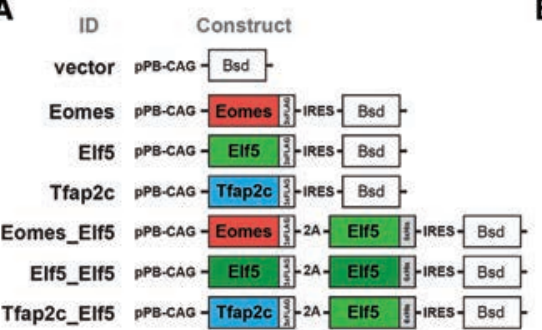

C

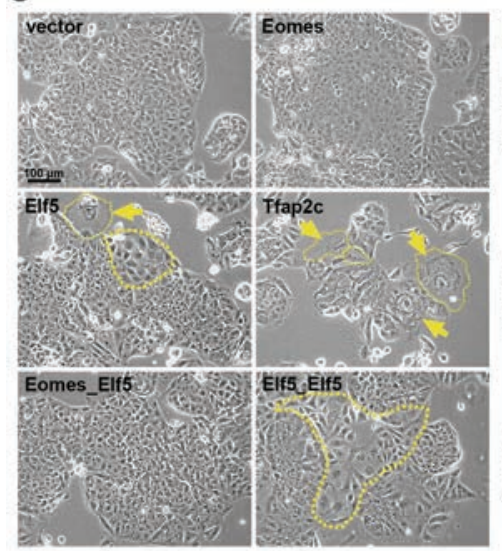

D
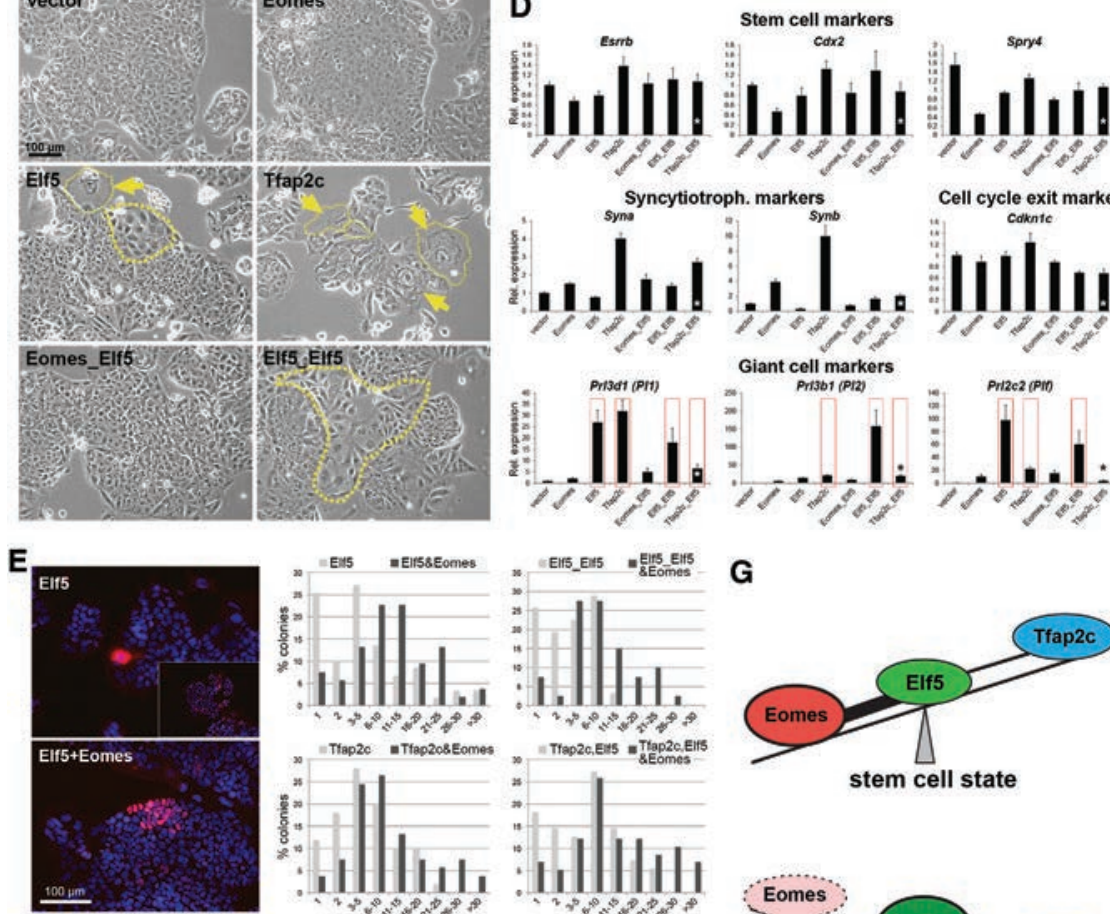

$\mathbf{F}$

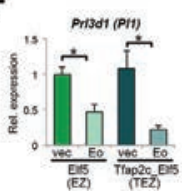

$B$
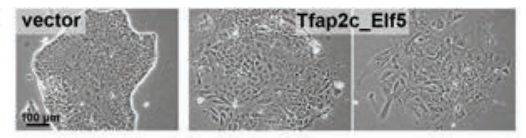

stem cell markers
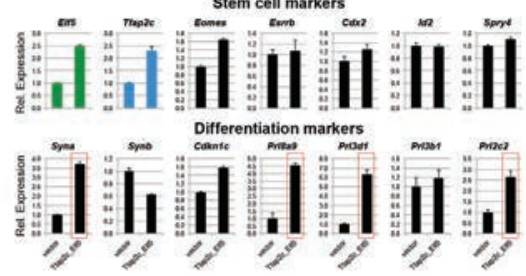
Syncytiotroph. markers
Cell cycle exit marker

G
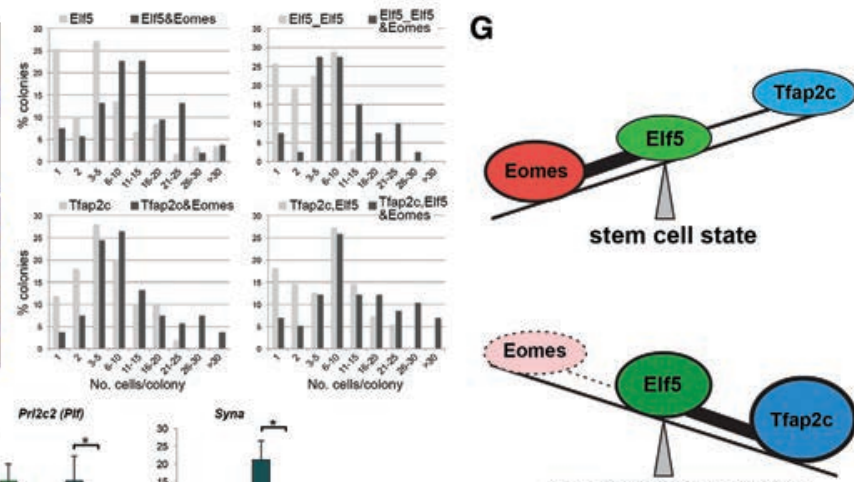

onset of differentiation

Figure 4. Stoichiometry-dependent effects of Eomes, Elf5, and Tfap2c. (A) Schematic diagram of expression constructs used to generate stable TSC lines. (B) Characteristics of Tfap2c_Elf5-overexpressing cells after short-term selection $17 \mathrm{~d}$ after transfection and $5 \mathrm{~d}$ after selection): Cells lose the tight epithelial morphology characteristic for TSCs in the stem cell state and up-regulate a series of differentiation markers, most notably those indicative of giant cell differentiation (Prl8a9, Prl3d1 $=$ P11; Prl3b1 $=$ P12; and Prl2c2 =Plf . Additional data on Tfap2c_Elf5-overexpressing cells are provided in Supplemental Figure S3. (C) Phase contrast images of representative colonies of the various stable TSC lines after $\sim 6 \mathrm{wk}$ in culture. Note the tight epithelial colony shape in Eomes-overexpressing TSCs (Eomes, also Eomes_ Elf5) and the high frequency of trophoblast giant cells (characterized by large cell and nuclear size) in Elf5-, Elf5_Elf5-, and Tfap2c-overexpressing cell lines. Examples of giant cells are highlighted by arrows, and cell dimensions are outlined by the thin dotted line. Larger areas of differentiating cells are highlighted by the thick yellow dashed line. The Tfap2c_Elf5 line is not shown, as it had largely lost transgene expression by this stage (see Supplemental Fig. S3D). (D) RTqPCR analysis of various TSC-specific and differentiation stage-specific marker genes in the stable cell lines shown in C. Note that the Tfap2c_Elf5 line marked with an asterisk had largely lost transgene expression, explaining the less pronounced up-regulation of trophoblast giant cell markers. $(E)$ (Co-)transfection experiments of the indicated expression constructs with or without Eomes. Immunofluorescence staining for Flag identifies transfected cells. Numbers of transfected cells per colony were counted $3 \mathrm{~d}$ after transfection for 40-60 separate colonies. Only cells with very low-level expression of Elf5 and/or Tfap2c form larger colonies. The presence of Eomes rescues the differentiation-promoting, anti-proliferative phenotype of Elf5 and/or Tfap2c overexpression. $(F)$ Transfection of Eomes into established Elf5 and Tfap2c_Elf5 cell lines reduces the enhanced expression of differentiation markers. $(G)$ Seesaw model of the function of Elf5 at the fulcrum between TSC self-renewal and differentiation. The stem cell state is characterized by preferential binding of Elf5 to Eomes in the presence of comparatively low Tfap2c amounts. Differentiation is triggered by an increase in Elf5 and Tfap2c levels and their predominant interaction.

differentiation-promoting effect could be quenched by concomitant overexpression of Eomes. This was evident in TSC lines carrying the Eomes_Elf5 construct but also upon cotransfection of Eomes with Elf5 and/or Tfap2c (Fig. 4E). Eomes expression reduced differentiation rates even in established Elf5 and Tfap2c_Elf5 cell lines (Fig. 4F).

To further assess this apparent dependence on protein stoichiometry, we performed knockdown experiments of Eomes in TSCs (Supplemental Fig. S4). Mirroring the relative shift in TF balance induced by Elf5 and Tfap2c overexpression, reduced Eomes levels also promoted TSC differentiation, characterized by down-regulation of TSC markers such as $C d \times 2$ and up-regulation of trophoblast giant cell and syncytiotrophoblast markers (Supplemental Fig. S4D). Importantly, we also showed that knockdown of Elf5 induced trophoblast differentiation, thus demonstrating that both too high and too low amounts of Elf5 interfered with TSC maintenance (Supplemental Fig. S4D,E). We compared the global gene expression changes in these loss-of-function and gain-of-function models to TSC differentiation induced by Fgf/CM withdrawal for 1 and $3 \mathrm{~d}$ (Supplemental Fig. S4F). This analysis confirmed 
that (1) Eomes-overexpressing cells are most similar to TSCs, (2) down-regulation of Eomes and Elf5 rapidly triggers significant differentiation, and (3) overexpression of Elf5 globally induces a state of early-onset differentiation, which corroborates the critical role of Elf5 levels precisely at the tipping point between TSC self-renewal and exit from the stem cell state.

Collectively, this careful dissection of TF levels revealed that a balanced expression of Eomes and Elf5 promotes the stem cell state, while proportionally higher Elf5 and Tfap2c levels trigger TSC differentiation (Fig. 4G).

\section{Dissecting the transcriptional networks established by Eomes, Elf5, and Tfap2c}

To identify genomic loci occupied by the various binary and ternary combinations between Eomes, Elf5, and Tfap2c, we performed ChIP-seq for Tfap2c and integrated these data with previously published Eomes and Elf5 ChIP-seq profiles in TSCs (Chuong et al. 2013). In total, we identified 1254 loci cobound by all three TFs, and 4254, 2035, and 766 sites cobound only by Elf5-Tfap2c, Elf5-Eomes, and Eomes-Tfap2c, respectively (Fig. 5A). These were distributed across the genome in a largely similar pattern (Supplemental Fig. S5A). Importantly, the numbers of observed combinatorial binding sites were highly statistically significant over co-occupancy expected at random, in particular for the triple, the Elf5-Tfap2c, and the Elf5-Eomes combinations (Fig. 5B). Interestingly, co-occupancy of Tfap2c with Sox2, another TF known to interact with Tfap2c in TSCs (Adachi et al. 2013), was confined to a set of sites largely distinct from those bound by Tfap2c and Elf5 (Fig. 5C). Overlap with Cdx2 was marginal (Supplemental Fig. S5B). These chromatin-binding patterns strongly support our functional and protein-protein interaction data of a concerted action of Eomes, Elf5, and Tfap2c in TSCs.

Eomes-Elf5-Tfap2c triple occupancy demarcates active TSC genes

Analysis of genes associated with the various co-occupied regions revealed that triply bound elements were highly enriched for TSC genes, including Eomes, Elf5, and Tfap2c themselves as well as others such as Bmp4, Cdh1, Fgfr2, Gata3, Sox2, Spry4, and Zic3 (Fig. 5D; Supplemental Table S2). This observation was corroborated by functional annotation (Genomic Regions Enrichment of Annotations Tool [GREAT]) analyses (McLean et al. 2010) in which triply bound genes were tightly linked with blastocyst formation and very early stages of trophoblast development (Fig. 5E; Supplemental Fig. S5C). In contrast, genes associated with Elf5-Tfap2c doubly bound loci such as Ccne1, Fzd5, Gcm1, Peg10, Prl2c2 (Plf), and Tfeb were overall enriched for processes related to trophoblast differentiation and placental development.

To further validate the correlation between triple-occupancy sites and expression of TSC genes, we performed transcriptomics (RNA-seq) on TSCs in the stem cell state and after $1 \mathrm{~d}$ of differentiation and integrated these data with the ChIP-seq peak maps. This analysis demonstrated that triply bound loci are associated with genes more highly expressed in stem cell conditions, underpinning the functional relevance of triple TF occupancy for transcriptional activation of stem cell genes (Fig. 5F).

\section{Dynamic shift toward TF-binding enrichment at Elf5-Tfap2c elements promotes differentiation}

Our next aim was to gain a refined view of the dynamics of TF occupancy in correlation with gene expression in TSCs and upon differentiation. Because Tfap2c levels are not limiting in either condition (in contrast to Eomes and Elf5), we used this TF as a readout of possible shifts in genome-wide binding patterns. The ChIP-seq data revealed that Tfap2c does not globally change genome occupancy after $1 \mathrm{~d}$ of differentiation; however, its relative enrichment levels shift between binding sites. Thus, triply bound genes (which are enriched for TSC expression) showed a higher Tfap2c-binding intensity in stem cell conditions. In contrast, with onset of differentiation, Tfap2c enrichment was increased at a larger number of Elf5-Tfap2c doubly bound and Tfap2c singly bound differentiationassociated genes (Fig. 6A,B).

The significance of this observation was further underpinned on multiple levels: The peak intensity of Tfap2c at Elf5-Tfap2c-cobound elements strongly correlated with expression levels and histone $\mathrm{H} 3 \mathrm{Lys} 4$ trimethylation (H3K4me3) (Rugg-Gunn et al. 2010) that demarcates active genes (Supplemental Fig. S5D). Thus, peaks with higher intensity in TSC conditions were associated with genes more highly expressed in TSCs. Conversely, peaks that became enriched upon differentiation correlated with genes up-regulated in 1-d-differentiated TSCs (Fig. 6C); these peaks were also preferentially located closer to the transcriptional start site (Supplemental Fig. S5E). We confirmed up-regulation of a number of genes associated with differentiation-linked Elf5-Tfap2c peaks upon $1 \mathrm{~d}$ of Fgf/CM withdrawal (Supplemental Fig. S6A). During subsequent stages of differentiation, the correlation between Elf5-Tfap2c sites and expression trailed off, as expected from an early function of Elf5 in the differentiation process (Supplemental Fig. S6B-D). This was corroborated by detailed cluster analysis of global gene expression changes, which revealed that genes whose expression changed only in later differentiation ( $3 \mathrm{~d}$ vs. $1 \mathrm{~d}$ ) were not associated with Elf5-Tfap2c-bound elements (Supplemental Fig. S6E). This finding strongly supported the notion that tipping the Elf5:Eomes ratio in favor of Elf5 (and Tfap2c) provides the initial trigger of differentiation. Notably, genes associated with Elf5-Tfap2c elements were also up-regulated in the Elf5- and Elf5_Elf5-overexpressing cells (Supplemental Fig. S6F).

Finally, to overcome the limitations of declining Elf5 levels with TSC differentiation, we established TSCs with doxycycline (dox)-inducible Elf5 expression and confirmed dose-dependent recruitment of Elf5 to Elf5-Tfap2c elements (Fig. 6D). Together, these data unraveled in 


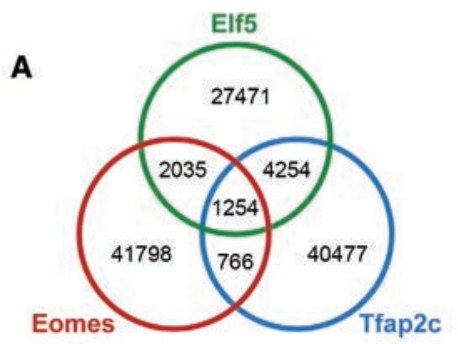

B

\begin{tabular}{|l|l|l|l|l|l|}
\hline Interaction & Obs & Exp & p value & adj p value & Enrichment \\
\hline Eli5/Eomes & 2035 & 465 & $5.1 \mathrm{E}-233$ & $1.3 \mathrm{E}-288$ & 4 \\
\hline Eli5/Tfap20 & 4254 & 334 & $0.0 \mathrm{E}+00$ & $0.0 \mathrm{E}+00$ & 13 \\
\hline Eomes/Tap2c & 766 & 378 & $7.0 \mathrm{E}-31$ & $1.2 \mathrm{E}-26$ & 2 \\
\hline Elr5/Eomes/Tap2c & 1254 & 3 & $0.0 \mathrm{E}+00$ & $0.0 \mathrm{E}+00$ & 418 \\
\hline
\end{tabular}
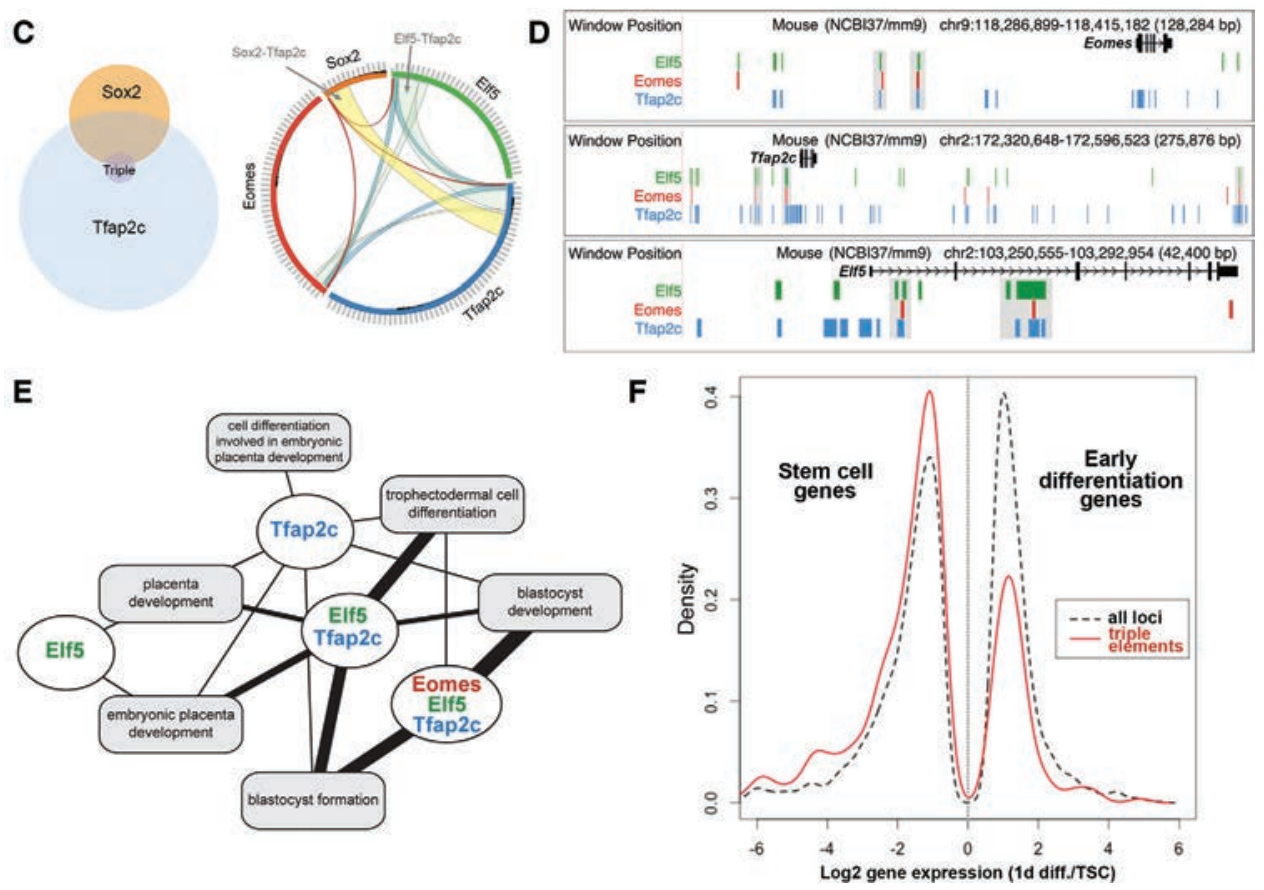

Figure 5. Elf5, Eomes, and Tfap2c triply bound regions are associated with active TSC genes. $(A)$ Venn diagram of the combinatorial ChIP-seq binding peaks between Elf5, Eomes, and Tfap2c in TSCs. (B) Bioinformatic analysis assessing the statistical significance of the occurrence of cobound elements over randomly expected. (C) Venn and chord diagrams depicting the genome-wide binding overlap of Tfap2c, Sox2, Eomes, and Elf5. Note that the Elf5-Tfap2c network is largely distinct from that established by the interaction between Tfap2c and Sox2. $(D)$ Genome browser views of the ChIP-seq peak distribution at the Eomes, Elf5, and Tfap2c loci. Triply bound elements are highlighted in gray. $(E)$ Functional annotation chart of the genes associated with single, double, and triple peaks. Only significant hits are shown; the TF combinations not shown did not exhibit significantly enriched functional annotations. The thickness of the connecting bars is indicative of the proportion of genes near bound elements that are annotated to the enriched term. Genes associated with triply bound regions are most strongly associated with early trophoblast development at the blastocyst stage, whereas Elf5-Tfap2c-associated genes are more strongly associated with later stages of trophoblast differentiation. $(F)$ Integration of RNA-seq data from TSCs grown in stem cell conditions and upon $1 \mathrm{~d}$ of differentiation with ChIP-seq peaks. The graph shows that genes more highly expressed in stem cell conditions are enriched for Elf5-Eomes-Tfap2c triply bound regions (red line) compared with all ChIP-seq loci.

intricate detail the stoichiometry-driven changes of the transcriptional networks regulating the onset of trophoblast differentiation.

\section{Differential TF-binding motif distribution underlies the shift toward a differentiation-promoting program}

To reveal the mechanism underlying these shifts in relative genome occupancy, we searched for TF motifs within the triple, double, and single ChIP-seq peaks. Globally, Tfap2c ChIP-seq elements were very tightly correlated with the Tfap2c consensus binding sequence, with 76\% of peaks overlapping the cognate Tfap2c motif. In stark contrast, the Tfap2c motif was significantly underrepre- sented at the 1254 triply bound sites, as only $405(32 \%)$ of them contained one (Fig. 6E). In comparison, the frequency of Elf5 motif recognition was similar or even enriched at triply bound sites over all Elf5 peaks. The Eomes motif did not lend itself to this same type of analysis, as it is too poorly defined. These findings implied that Tfap $2 \mathrm{c}$ binding to triple elements is mediated through its physical association with Elf5 and Eomes. In contrast, Tfap2c peaks that increased with differentiation harbored the cognate Tfap2c motif.

Overall, these findings provide a molecular mechanism for how the same TFs can orchestrate both self-renewal and differentiation-promoting transcriptional programs through a stoichiometry-sensitive shift in function of a 


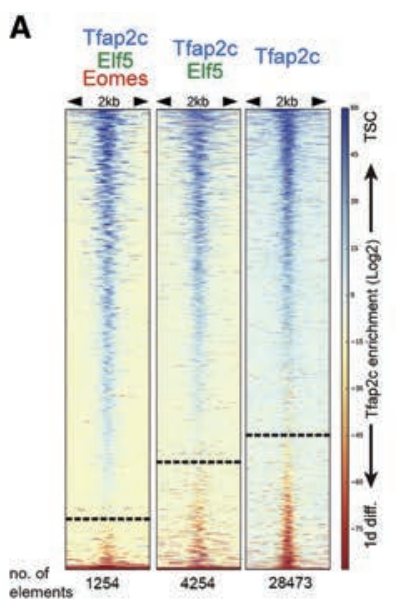

B

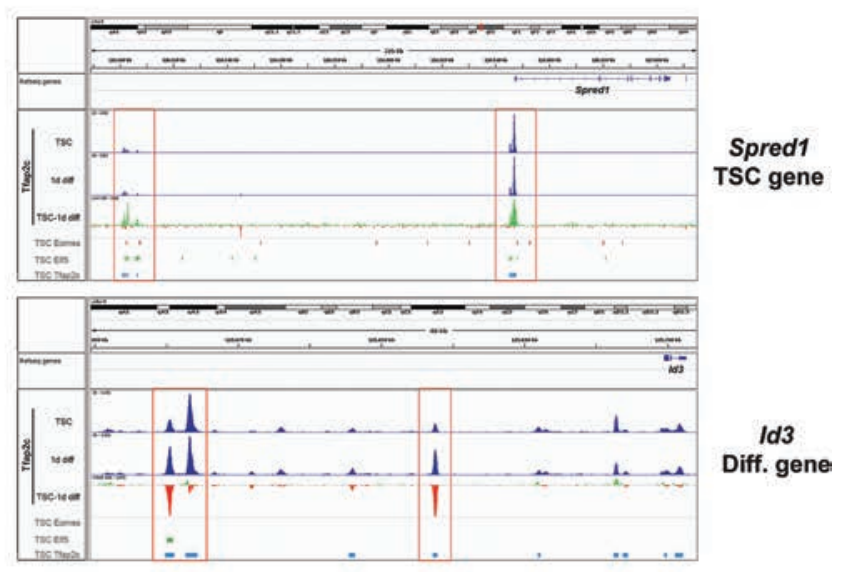

D

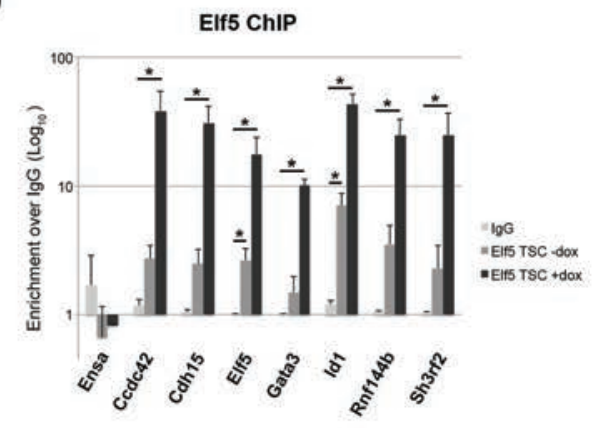

$\mathbf{E}$

\begin{tabular}{c|c|c|c|c|} 
& \multicolumn{2}{|c|}{ Tfap2c } & \multicolumn{2}{c|}{ Elf5 } \\
\hline all peaks & $76.1 \%$ & $23.9 \%$ & $41.1 \%$ & $58.9 \%$ \\
\hline $\begin{array}{l}\text { ternary } \\
\text { peaks }\end{array}$ & $\begin{array}{c}32.3 \% \\
(p<0.001)\end{array}$ & $67.7 \%$ & $51.6 \%$ & $48.4 \%$ \\
\hline & motif & $\begin{array}{c}\text { no } \\
\text { motif }\end{array}$ & motif & $\begin{array}{c}\text { no } \\
\text { motif }\end{array}$
\end{tabular}

F Trophoblast stem cell state

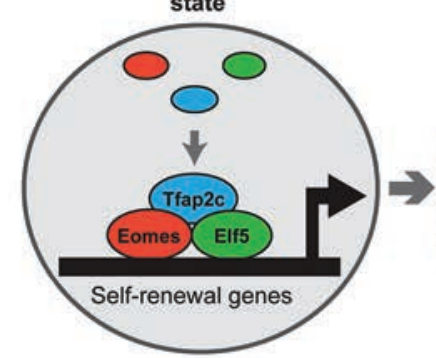

Onset of differentiation

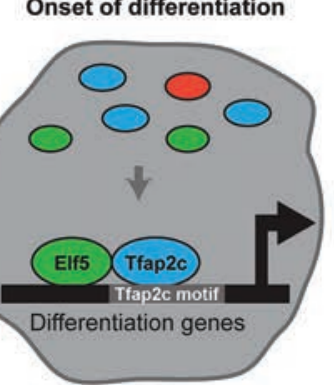

Figure 6. Elevated Elf5 and Tfap2c levels trigger TSC differentiation. (A) Differential ChIP-seq binding enrichment of Tfap2c in TSCs and upon $1 \mathrm{~d}$ of differentiation. The 1254 triply bound elements predominantly have a higher Tfap2c abundance in TSCs, indicated by the blue shading of elements. By comparison, a significantly larger proportion of the 4254 Elf5-Tfap2c double and 28,473 (intergenic) Tfap2c single peaks exhibit a higher Tfap2c abundance upon differentiation, indicated by the red-shaded elements (highlighted by the dashed lines). Please note that, since this analysis is based on the differential enrichment of Tfap2c between TSCs and 1-d-differentiated cells, only peak combinations with Tfap2c can be analyzed. (B) Example browser views of the differential Tfap2c peak enrichment in TSCs grown in stem cell conditions and upon $1 \mathrm{~d}$ of differentiation. Tfap $2 \mathrm{c}$ binding is higher in TSC conditions at triply bound sites at genes more highly expressed in TSCs such as Spred1; conversely, Tfap2c binding is enriched at Tfap2c singly and Elf5-Tfap2c doubly occupied sites in differentiation conditions surrounding genes up-regulated upon differentiation, such as Id3. (C) Correlation of differential Tfap2c ChIPseq peak binding intensity at Elf5-Tfap2c elements with expression levels of the associated genes. Higher enrichment of Tfap2c correlates with elevated gene expression both for TSC genes in stem cell conditions (left of vertical dotted line) and at genes up-regulated with differentiation in differentiation-promoting conditions (right of vertical dotted line). ( $D$ ) ChIP of Elf5 followed by qPCR for Elf5-Tfap2c target loci in TSCs that harbor a doxycycline (dox)-inducible Elf5 expression construct. Elf5 recruitment to these sites is increased upon Elf5 overexpression (Elf5 TSC + dox). $\left(^{*}\right) P<0.05$. (E) Differential distribution of Tfap2c motifs: Globally, Tfap2c ChIP-seq peaks overlap extremely closely with the Tfap $2 \mathrm{c}$ consensus binding sequence, with $76 \%$ of peaks positioned over the cognate motif. In contrast, triply bound sites are significantly depleted for the cognate motif, with only $32 \%$ of peaks overlapping the consensus sequence. Significance was calculated with a $\chi^{2}$ test with Yates' correction; the odds ratio is 6.7. The consensus motif for Elf5 is less tightly defined, with, globally, $41 \%$ of peaks overlapping the cognate sequence; this is increased to $52 \%$ at triply bound elements. (F) Model of the stoichiometry-dependent functions of Elf5, Eomes, and Tfap2c in fine-tuning the balance between TSC self-renewal and commitment to differentiate. 
small group of interacting TFs centered on Elf5 acting as a cell fate switch (Fig. 6F).

\section{Discussion}

Insights into the transcriptional networks required to maintain the self-renewal state of TSCs have largely relied on the analysis of mouse mutants and the inability to derive TSCs from them. These studies have revealed a number of TFs that are essential to establish and maintain TSCs, including Eomes, Elf5, and Tfap2c (Russ et al. 2000; Donnison et al. 2005; Kuckenberg et al. 2010). Here, we show that these same three TFs physically bind to each other to form multimeric TF complexes and co-occupy trophoblast genes, including themselves, driving their expression. In this sense, the self-renewal network of TSCs shares important mechanistic features with pluripotency circuits in ESCs not only in terms of mutual self-reinforcement but also as far as protein-protein interactions and genomic cobinding are concerned.

In addition to the identification of these core trophoblast TF interactomes and the networks that they establish, our findings add another important dimension to the understanding of TSC regulation: Not only are certain TFs essential for TSC self-renewal, but their proportional abundance is equally critical to maintain the stem cell state. We show that precise levels of Elf5 determine the balance between TSC self-renewal and onset of differentiation. Loss but also overexpression of Elf5 result in depletion of the proliferative stem cell compartment in vitro and in vivo and embryonic lethality around mid-gestation (Donnison et al. 2005). These data bear a strong resemblance to Oct4 as a key node of the pluripotency network in ESCs, whose fine-tuned expression levels control both the entry into and the exit from naïve pluripotency (Radzisheuskaya et al. 2013). Complete depletion of Oct4 causes differentiation toward trophoblast-like cells, but, conversely, high Oct4 levels are also required for differentiation into embryonic lineages and the germline, albeit the mechanism underlying this sensitivity to expression levels remains unknown (Niwa et al. 2000; Radzisheuskaya et al. 2013). Our insights reveal an analogous finely tuned balance for Elf5 in TSCs and place Elf5 at the center of the transcriptional networks governing self-renewal as well as differentiation in TSCs.

To gain a better molecular understanding of Elf5's function in this circuit, we analyzed its interacting partners in TSCs using mass spectrometry. In addition to Eomes and Tfap2c, we identified other Elf5 interactors with known roles in trophoblast and placental development, including Grhl2 (Walentin et al. 2015) and Bptf (Goller et al. 2008). Our data showed that Elf5 associates with components of both activating (e.g., Bptf and Chd7) and repressive (Sin3 and Polycomb) chromatin complexes, indicating its role in both gene activation and repression. It is noteworthy that the Elf5 interactome did not include other critical TSC TFs such as Cdx2 or Esrrb, and we did not identify Elf5 associated with Cdx2 or Esrrb in the reciprocal interactomes (Latos et al. 2015). Together with the minimal genomic binding overlap between $\mathrm{Cdx} 2$ and Elf5, it appears that these TFs function in mostly parallel circuits to maintain self-renewal of TSCs, except at retroviral elements where they have a joint function /Chuong et al. 2013).

Our detailed dissection of the Eomes-Elf5-Tfap2c network in TSCs shows that the relative levels of these three TFs determine preferential interaction partners and, consequently, binding and activation of separate sets of genes with opposing functions. The presence of Eomes in at least equal stoichiometric quantities ensures occupancy at triply bound genomic sites and expression of the associated TSC genes. This joint genome occupancy is facilitated by formation of a ternary TF complex. However, when Elf5 and Tfap2c levels exceed those of Eomes, they become enriched at Elf5-Tfap2c double (and single) sites, triggering the exit from self-renewal through activation of a differentiation-promoting program. Mechanistically, this is enabled by the differential distribution of the Tfap2c consensus motif; while triply occupied sites are depleted for this sequence element, and Tfap $2 \mathrm{c}$ binding is indirect through association with Elf5 and Eomes, recruitment of Elf5:Tfap2c to sites containing the cognate Tfap2c motif is favored as Elf5 and Tfap2c protein levels increase (Fig. 6F).

Overall, our data reveal an intricate balance between mutually interacting TFs that is critical to determine stem cell self-renewal versus differentiation in the trophoblast compartment. This sensitivity of TSCs to precise TF stoichiometries is a likely reason for the significant spontaneous differentiation rates commonly observed in TSC cultures. It may also explain why reprogramming strategies based on overexpression or activation of selected TFs specifically between ESCs and TSCs have proved difficult and tend to result in loss of self-renewal potential and terminal differentiation (Niwa et al. 2000, 2005; Ralston et al. 2010; Cambuli et al. 2014). In the bigger picture, this precarious balance might explain why derivation and maintenance of TSCs are difficult and have often proved inefficient or even unsuccessful from other species, in particular humans (Kunath et al. 2014). Gaining detailed insights into the precise regulation of transcriptional networks governing trophoblast development is therefore essential to achieve these goals.

\section{Materials and methods}

Generation of trophoblast-specific Elf5-overexpressing conceptuses

Generation of lentivirally mediated trophoblast-specific overexpression was performed largely as described previously (Okada et al. 2007; Morioka et al. 2009). Briefly, B6D2F1 female mice were superovulated by intraperitoneal injection of $5 \mathrm{U}$ of pregnant mare's serum gonadotropin followed by $5 \mathrm{U}$ of human chorionic gonadotropin $48 \mathrm{~h}$ later and then mated with B6D2F1 males. Two-cell stage embryos were collected from females 1.5 $\mathrm{d}$ after copulation and then incubated for $2 \mathrm{~d}$ to obtain blastocysts. Zona-free blastocysts were prepared by treatment with acidic Tyrode's solution. Blastocysts were then incubated in groups of four in $4 \mu \mathrm{L}$ of medium containing lentiviral vector 
(pCAG-EGFP or p|CAG-Elf5; 1000 ng of p24 per mL) for $5 \mathrm{~h}$. Transduced blastocysts were implanted into the uteri of E2.5 pseudopregnant females. Litters were dissected at the indicated days of development.

\section{Stem cell culture}

TSC lines EGFP, Rs26, and "CTRL WT" (a kind gift of the Rossant [Toronto, Canada] and Okano [Kobe, Japan] laboratories) were cultured as described previously (Tanaka et al. 1998). Further manipulations were performed with piggyBac vectors containing the complete ORFs of TFs as indicated. TFs were cloned and sequence-verified. All transformations to yield stable cell lines were performed in the TS-EGFP cell line, which exhibits proven placental contribution competence (Tanaka et al. 1998; Cambuli et al. 2014).

\section{Histological analyses}

Standard immunohistochemistry was performed on 7- $\mu \mathrm{m}$ sections as detailed in the Supplemental Material. Images were taken on an Olympus BX41 or BX61 epifluorescence microscope or a Zeiss LSM 780 confocal microscope.

For RNA in situ hybridizations, linearized plasmids containing cDNA inserts from Plf and Tpbpa cDNAs were used to generate digoxigenin (DIG)-labeled riboprobes according to the manufacturer's instructions (Roche). Hybridizations were carried out overnight at $52^{\circ} \mathrm{C}$ using standard procedures. Signals were detected with anti-DIG-alkaline phosphatase-conjugated antibody (Roche), and staining was performed overnight using NBT and BCIP reagents (Promega). Sections were counterstained with nuclear Fast Red (Sigma).

\section{Coimmunoprecipitation}

TS-EGFP cells were transfected with a piggyBac-CAG-Avi-Elf53xFlag-Ires-Neo, piggyBac-CAG-Eomes-3xFlag-Ires-Bsd, or piggyBac-CAG-Tfap2c-3xFlag-Ires-Bsd construct containing the Elf5, Eomes, Tfap2c coding sequences, respectively, or empty vector alone using Lipofectamine 2000 (Invitrogen). Cells were selected with G418 or Blasticidin S and expanded to $1015-\mathrm{cm}$ dishes. Cells were washed in PBS, harvested, and used for immunoprecipitation exactly as described previously (van den Berg et al. 2010). Precise details are given in the Supplemental Material. Eluates were pooled and analyzed by mass spectrometry or Western blot.

\section{Mass spectrometry}

Immunoprecipitated proteins from three biological replicates each of Elf5 and vector transfected TSCs were analyzed as before (Webster and Oxley 2009; Latos et al. 2015). Mass spectrometric data were processed using Proteome Discoverer version 1.4 (Thermo Scientific) and searched against the mouse entries in UniProt 2013.09 and a database of common contaminants using Mascot version 2.3 (Matrix Science), and the results were imported into Scaffold version 3.6 (Proteome Software, Inc.). With protein/peptide thresholds of $50 \% / 0 \%$ and a minimum of two peptides, a total of 694 proteins was reported across the six samples, with a calculated protein false discovery rate of $0.0 \%$. After further filtering, (proteins identified in at least two of the three replicates and having average spectral count ratios relative to the controls $>2$ ), 109 proteins remained, which are shown in Supplemental Table S1.

\section{$R T-q P C R s$}

Total RNA was extracted using TRI reagent (Sigma, T9424) according to the manufacturer's instructions, and any potential DNA contamination was removed by treatment the Turbo DNA-free kit (Life Technologies, AM1907) according to the manufacturer's instructions. One micrgram to $2 \mu \mathrm{g}$ of total RNA was used for cDNA synthesis with RevertAid H-minus M-MuLV revert transcriptase (Fermentas, EP0451) and random hexamers (Promega, C118A).

qPCR was performed using SYBR Green Jump Start Taq Ready mix (Sigma, S4438) on a Bio-Rad CFX96 thermocycler.

\section{Western blotting}

Whole-cell extracts were prepared with transgenic buffer $(20 \mathrm{mM}$ Tris- $\mathrm{HCl}$ at $\mathrm{pH} 7.5,137 \mathrm{mM} \mathrm{NaCl}, 1 \mathrm{mM}$ EGTA, 1\% Triton $\mathrm{X}-100,10 \%$ glycerol, $1.5 \mathrm{mM} \mathrm{MgCl} 2$ ) supplemented with protease inhibitor cocktail (Sigma, P2714). Nuclear extracts were prepared as described previously (van den Berg et al. 2010). Western blotting was carried out according to a standard protocol (see the Supplemental Material). The antibodies used were anti-Flag (Sigma, F1804), anti-Elf5 (Santa Cruz Biotechnology, sc-9645), anti-Eomes (Abcam, ab23345; R\&D Systems, MAB 6166), antiTfap2c (R\&D System, AF5059), anti-tubulin (Abcam ab6160), and ImmunoCruz IP/WB Optima System C (sc-45040), E (sc-45042), or A (sc-45038).

\section{ChIP-seq}

Immunoprecipitations were carried out as described (Tavares et al. 2012; Latos et al. 2015) and are detailed in the Supplemental Material. DNA from four immunoprecipitations was pooled for each library generated with the NEB Next DNA library preparation master mix (New England Biolabs, E6040) according to the manufacturer's instructions. Libraries were amplified using 18 PCR cycles, purified using Agencourt AMPure XP SPRI beads (Beckman Coulter, A63881), and size-selected on an agarose gel. DNA was extracted using the QiaQuick gel extraction kit (Qiagen), and its concentration was determined using the KAPA Illumina SYBR Universal Lid Q kit (KAPA Biosystems, KK4824) and Bioanalyzer 2100 system (Agilent). Libraries were sequenced on an Illumina HiSeq 2500 sequencer.

\section{Global expression profiling}

RNA-seq was performed on chorion and trophoblast tissue material recovered from paraffin sections according to the FFPE miRNeasy kit (Qiagen, 217504). rRNA depletion was performed using the NEB Next kit (New England Biolabs, E6310S). For RNA-seq from TSCs, total RNA was prepared using TRI reagent (Sigma, T9424) followed by DNase treatment using the Turbo DNA-free kit (Life Technologies, AM1907). mRNA was isolated from 150-240 ng of total DNA-free RNA using the Dynabeads mRNA purification kit (Life Technologies, 61006). Indexed, strand-specific libraries were prepared using the ScriptSeq version 2 RNA-seq library preparation kit (Epicentre, SSV21106). Libraries were quantified using both the KAPA library quantification kit (KAPA Biosystems, KK4824) and Bioanalyzer 2100 system (Agilent). Indexed libraries were pooled and sequenced with a 100-base-pair single-end protocol on an Illumina HiSeq 2500 sequencer. Raw FastQ data were mapped to the Mus musculus NCBIM37 genome assembly using TopHat version 2.0.12 (Kim et al. 2013). Data were analyzed using DESeq and the RNA-seq 
quantitation pipeline in SeqMonk software (http://www. bioinformatics.babraham.ac.uk).

\section{Bioinformatic analysis}

Raw reads were aligned to mouse genome build mm9 using Bowtie, and peak calling was performed with MACS2 with default parameters (Zhang et al. 2008). To identify overlapping elements from different ChIP experiments, BED files of ChIP elements were compared using the BedTools (Quinlan and Hall 2010) function multiinter with the cluster option to reduce redundant interactions to the highest-order interactions. Overlapping regions required a $50 \%$ overlap to be included. To model the all by all possible interactions, we used the shuffle function in the R library ChIPseeker (Yu et al. 2015) to make randomized BED files of each ChIP experiment. We then used the multiinter function of BedTools to generate a table of expected interactions of the randomized data. This was repeated 10,000 times to build average models. We then tested our observed data against the random models using a Poisson exact test. $P$-values were adjusted for multiple testing $(n=175,123$ to account the total number of all ChIP elements involved in the analysis).

Annotation of binding sites according to genomic features was performed by overlapping the sites with Ensembl version 77 annotations. Motif analysis was performed with MEME-chip (Machanick and Bailey 2011) using the JASPAR_CORE_2014_ vertebrates database, searching for zero or one occurrences of the motif per peaks and with a maximum number of motifs discovered by MEME of 12 . Additional details are provided in the Supplemental Material; all high-throughput sequencing data were deposited in publicly accessible repositories (accession no. PRJNA298763).

\section{Acknowledgments}

We are grateful to Kristina Tabbada for Illumina high-throughput sequencing, Judith Webster for assistance with mass spectrometry experiments, Anne Segonds-Pichon for expert statistical help, and Oliver Tam, Simon Andrews, and Felix Krueger for help with the bioinformatic analysis. This work was supported by a Next-Generation Fellowship (awarded to P.A.L. by the Centre for Trophoblast Research, University of Cambridge), the Biotechnology and Biological Sciences Research Council (BBSRC), and the Wellcome Trust. B.J.C. is supported by a Canada Research Chair and an operating grant from the Canadian Institutes of Health Research.

\section{References}

Adachi K, Nikaido I, Ohta H, Ohtsuka S, Ura H, Kadota M, Wakayama T, Ueda HR, Niwa H. 2013. Context-dependent wiring of Sox2 regulatory networks for self-renewal of embryonic and trophoblast stem cells. Mol Cell 52: 380-392.

Auman HJ, Nottoli T, Lakiza O, Winger Q, Donaldson S, Williams T. 2002. Transcription factor AP- $2 \gamma$ is essential in the extra-embryonic lineages for early postimplantation development. Development 129: 2733-2747.

Avilion AA, Nicolis SK, Pevny LH, Perez L, Vivian N, LovellBadge R. 2003. Multipotent cell lineages in early mouse development depend on SOX2 function. Genes Dev 17: 126-140.

Cambuli F, Murray A, Dean W, Dudzinska D, Krueger F, Andrews S, Senner CE, Cook SJ, Hemberger M. 2014. Epigenetic mem- ory of the first cell fate decision prevents complete ES cell reprogramming into trophoblast. Nat Commun 5: 5538.

Carey BW, Markoulaki S, Hanna JH, Faddah DA, Buganim Y, Kim J, Ganz K, Steine EJ, Cassady JP, Creyghton MP, et al. 2011. Reprogramming factor stoichiometry influences the epigenetic state and biological properties of induced pluripotent stem cells. Cell Stem Cell 9: 588-598.

Chuong EB, Rumi MA, Soares MJ, Baker JC. 2013. Endogenous retroviruses function as species-specific enhancer elements in the placenta. Nat Genet 45: 325-329.

Donnison M, Beaton A, Davey HW, Broadhurst R, L'Huillier P, Pfeffer PL. 2005. Loss of the extraembryonic ectoderm in Elf5 mutants leads to defects in embryonic patterning. Development 132: 2299-2308.

Donnison M, Broadhurst R, Pfeffer PL. 2015. Elf5 and Ets2 maintain the mouse extraembryonic ectoderm in a dosage dependent synergistic manner. Dev Biol 397: 77-88.

Georgiades P, Cox B, Gertsenstein M, Chawengsaksophak K, Rossant J. 2007. Trophoblast-specific gene manipulation using lentivirus-based vectors. Biotechniques 42: 317-318.

Goller T, Vauti F, Ramasamy S, Arnold HH. 2008. Transcriptional regulator $\mathrm{BPTF} / \mathrm{FAC1}$ is essential for trophoblast differentiation during early mouse development. Mol Cell Biol 28: 6819-6827.

Home P, Ray S, Dutta D, Bronshteyn I, Larson M, Paul S. 2009. GATA3 is selectively expressed in the trophectoderm of peri-implantation embryo and directly regulates $\mathrm{Cdx} 2$ gene expression. J Biol Chem 284: 28729-28737.

Kidder BL, Palmer S. 2010. Examination of transcriptional networks reveals an important role for TCFAP2C, SMARCA4, and EOMES in trophoblast stem cell maintenance. Genome Res 20: 458-472.

Kim D, Pertea G, Trapnell C, Pimentel H, Kelley R, Salzberg SL. 2013. TopHat2: accurate alignment of transcriptomes in the presence of insertions, deletions and gene fusions. Genome Biol 14: R36.

Kuckenberg P, Buhl S, Woynecki T, van Furden B, Tolkunova E, Seiffe F, Moser M, Tomilin A, Winterhager E, Schorle H. 2010. The transcription factor TCFAP2C/AP- $2 \gamma$ cooperates with CDX2 to maintain trophectoderm formation. Mol Cell Biol 30: 3310-3320.

Kunath T, Yamanaka Y, Detmar J, MacPhee D, Caniggia I, Rossant J, Jurisicova A. 2014. Developmental differences in the expression of FGF receptors between human and mouse embryos. Placenta 35: 1079-1088.

Latos PA, Goncalves A, Oxley D, Mohammed H, Turro E, Hemberger M. 2015. Fgf and Esrrb integrate epigenetic and transcriptional networks that regulate self-renewal of trophoblast stem cells. Nat Commun 6: 7776.

Machanick P, Bailey TL. 2011. MEME-ChIP: motif analysis of large DNA datasets. Bioinformatics 27: 1696-1697.

Martello G, Smith A. 2014. The nature of embryonic stem cells. Annu Rev Cell Dev Biol 30: 647-675.

McLean CY, Bristor D, Hiller M, Clarke SL, Schaar BT, Lowe CB, Wenger AM, Bejerano G. 2010. GREAT improves functional interpretation of cis-regulatory regions. Nat Biotechnol 28: 495-501.

Morioka Y, Isotani A, Oshima RG, Okabe M, Ikawa M. 2009. Placenta-specific gene activation and inactivation using integrase-defective lentiviral vectors with the Cre/LoxP system. Genesis 47: 793-798.

Ng RK, Dean W, Dawson C, Lucifero D, Madeja Z, Reik W, Hemberger M. 2008. Epigenetic restriction of embryonic cell lineage fate by methylation of Elf5. Nat Cell Biol 10: 1280-1290. 
Nichols J, Smith A. 2012. Pluripotency in the embryo and in culture. Cold Spring Harb Perspect Biol 4: a008128.

Nishioka N, Yamamoto S, Kiyonari H, Sato H, Sawada A, Ota M, Nakao K, Sasaki H. 2008. Tead4 is required for specification of trophectoderm in pre-implantation mouse embryos. Mech Dev 125: 270-283.

Niwa H, Miyazaki J, Smith AG. 2000. Quantitative expression of Oct-3/4 defines differentiation, dedifferentiation or self-renewal of ES cells. Nat Genet 24: 372-376.

Niwa H, Toyooka Y, Shimosato D, Strumpf D, Takahashi K, Yagi $\mathrm{R}$, Rossant J. 2005. Interaction between Oct3/4 and Cdx2 determines trophectoderm differentiation. Cell 123: 917-929.

Okada Y, Ueshin Y, Isotani A, Saito-Fujita T, Nakashima H, Kimura K, Mizoguchi A, Oh-Hora M, Mori Y, Ogata M, et al. 2007. Complementation of placental defects and embryonic lethality by trophoblast-specific lentiviral gene transfer. Nat Biotechnol 25: 233-237.

Quinlan AR, Hall IM. 2010. BedTools: a flexible suite of utilities for comparing genomic features. Bioinformatics 26: 841-842.

Radzisheuskaya A, Chia Gle B, dos Santos RL, Theunissen TW, Castro LF, Nichols J, Silva JC. 2013. A defined Oct4 level governs cell state transitions of pluripotency entry and differentiation into all embryonic lineages. Nat Cell Biol 15: 579-590.

Ralston A, Rossant J. 2008. Cdx2 acts downstream of cell polarization to cell-autonomously promote trophectoderm fate in the early mouse embryo. Dev Biol 313: 614-629.

Ralston A, Cox BJ, Nishioka N, Sasaki H, Chea E, Rugg-Gunn P, Guo G, Robson P, Draper JS, Rossant J. 2010. Gata3 regulates trophoblast development downstream of Tead4 and in parallel to Cdx2. Development 137: 395-403.

Rugg-Gunn PJ, Cox BJ, Ralston A, Rossant J. 2010. Distinct histone modifications in stem cell lines and tissue lineages from the early mouse embryo. Proc Natl Acad Sci 107: 10783-10790.

Russ AP, Wattler S, Colledge WH, Aparicio SA, Carlton MB, Pearce JJ, Barton SC, Surani MA, Ryan K, Nehls MC, et al. 2000. Eomesodermin is required for mouse trophoblast development and mesoderm formation. Nature 404: 95-99.

Shimokawa K, Kimura-Yoshida C, Nagai N, Mukai K, Matsubara K, Watanabe H, Matsuda Y, Mochida K, Matsuo I. 2011. Cell surface heparan sulfate chains regulate local reception of FGF signaling in the mouse embryo. Dev Cell 21: 257-272.

Strumpf D, Mao CA, Yamanaka Y, Ralston A, Chawengsaksophak K, Beck F, Rossant J. 2005. Cdx2 is required for correct cell fate specification and differentiation of trophectoderm in the mouse blastocyst. Development 132: 2093-2102.

Tanaka S, Kunath T, Hadjantonakis AK, Nagy A, Rossant J. 1998. Promotion of trophoblast stem cell proliferation by FGF4. Science 282: 2072-2075.
Tavares L, Dimitrova E, Oxley D, Webster J, Poot R, Demmers J, Bezstarosti K, Taylor S, Ura H, Koide H, et al. 2012. RYBPPRC1 complexes mediate $\mathrm{H} 2 \mathrm{~A}$ ubiquitylation at polycomb target sites independently of PRC2 and H3K27me3. Cell 148: 664-678.

Tremblay GB, Kunath T, Bergeron D, Lapointe L, Champigny C, Bader JA, Rossant J, Giguere V. 2001. Diethylstilbestrol regulates trophoblast stem cell differentiation as a ligand of orphan nuclear receptor ERR $\beta$. Genes Dev 15: 833-838.

van den Berg DL, Snoek T, Mullin NP, Yates A, Bezstarosti K, Demmers J, Chambers I, Poot RA. 2010. An Oct4-centered protein interaction network in embryonic stem cells. Cell Stem Cell 6: 369-381.

Walentin K, Hinze C, Werth M, Haase N, Varma S, Morell R, Aue A, Potschke E, Warburton D, Qiu A, et al. 2015. A Grhl2-dependent gene network controls trophoblast branching morphogenesis. Development 142: 1125-1136.

Webster J, Oxley D. 2009. Protein identification by peptide mass fingerprinting using MALDI-TOF mass spectrometry. In The protein protocols handbook (ed. Walker JM), pp. 1117-1129. Humana Press, New York.

Werling U, Schorle H. 2002. Transcription factor gene AP-2 $\gamma$ essential for early murine development. Mol Cell Biol 22: 3149-3156.

Yagi R, Kohn MJ, Karavanova I, Kaneko KJ, Vullhorst D, DePamphilis ML, Buonanno A. 2007. Transcription factor TEAD4 specifies the trophectoderm lineage at the beginning of mammalian development. Development 134: 3827-3836.

Yamamoto H, Flannery ML, Kupriyanov S, Pearce J, McKercher SR, Henkel GW, Maki RA, Werb Z, Oshima RG. 1998. Defective trophoblast function in mice with a targeted mutation of Ets2. Genes Dev 12: 1315-1326.

Yang J, Chai L, Fowles TC, Alipio Z, Xu D, Fink LM, Ward DC, Ma Y. 2008. Genome-wide analysis reveals Sall4 to be a major regulator of pluripotency in murine-embryonic stem cells. Proc Natl Acad Sci 105: 19756-19761.

Yu G, Wang LG, He QY. 2015. ChIPseeker: an R/Bioconductor package for ChIP peak annotation, comparison and visualization. Bioinformatics 31: 2382-2383.

Zhang J, Tam WL, Tong GQ, Wu Q, Chan HY, Soh BS, Lou Y, Yang J, Ma Y, Chai L, et al. 2006. Sall4 modulates embryonic stem cell pluripotency and early embryonic development by the transcriptional regulation of Pou5f1. Nat Cell Biol 8: 1114-1123.

Zhang Y, Liu T, Meyer CA, Eeckhoute J, Johnson DS, Bernstein BE, Nusbaum C, Myers RM, Brown M, Li W, et al. 2008. Model-based analysis of ChIP-seq (MACS). Genome Biol 9: R137. 


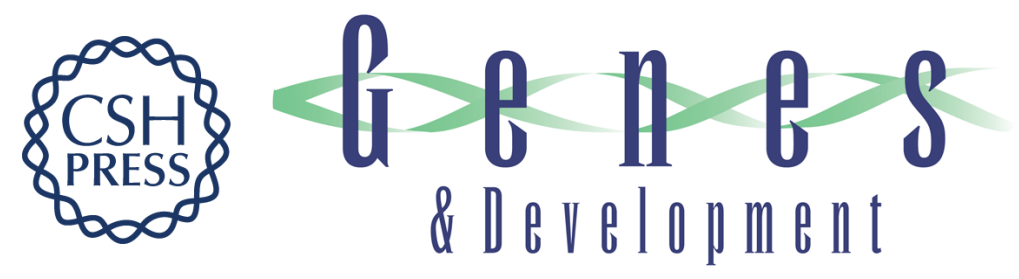

\section{Elf5-centered transcription factor hub controls trophoblast stem cell self-renewal and differentiation through stoichiometry-sensitive shifts in target gene networks}

Paulina A. Latos, Arnold R. Sienerth, Alexander Murray, et al.

Genes Dev. 2015, 29: originally published online November 19, 2015

Access the most recent version at doi:10.1101/gad.268821.115

Supplemental Material

References

Creative

Commons

License

Email Alerting

Service
http://genesdev.cshlp.org/content/suppl/2015/11/18/gad.268821.115.DC1

This article cites 46 articles, 18 of which can be accessed free at: http://genesdev.cshlp.org/content/29/23/2435.full.html\#ref-list-1

This article, published in Genes \& Development, is available under a Creative Commons License (Attribution-NonCommercial 4.0 International), as described at http://creativecommons.org/licenses/by-nc/4.0/.

Receive free email alerts when new articles cite this article - sign up in the box at the top right corner of the article or click here.

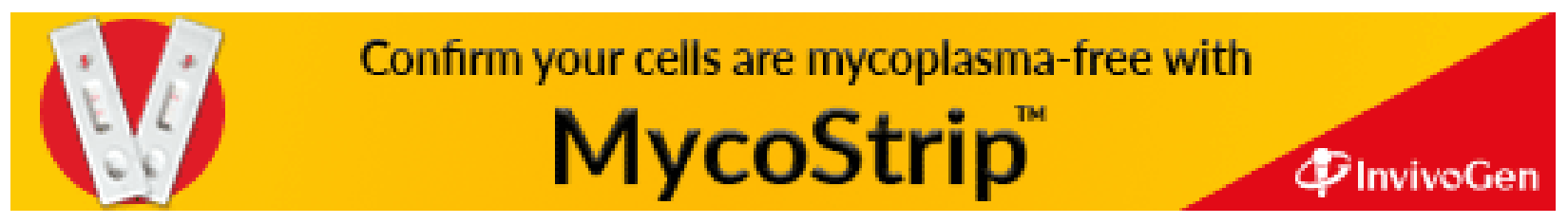

